\section{A Debatable Drug Trial System}

\section{Ashish Chauhan*}

National Institute of Pharmaceutical Education and Research, Mohali, Punjab, India

In drug delivery system, patients are often given medicines or drugs with no active ingredients and yet still report positive effects. This is called as the placebo effect. The word placebo refers to please in Latin and the medical definition of a placebo is a treatment that has no medicinal value or no pharmacological effects. Sometimes, referred to as 'sugar pills', placebos are usually used in drug trials to judge the competence and effectiveness of new medicines or drug moiety.

Surprisingly, placebos administered have shown to provide relief from the symptoms of illnesses such as asthma, depression, diabetes, epilepsy, migraine, wraths, schizophrenia and ulcers. Up to $30 \%$ of patients experience a reduction of symptoms due to placebo treatments.

\section{Types of Placebos}

There have been studies into the type of placebos that have the best effect. The color and size of tablets and capsules have been experimented but the results are commonly inconclusive. It does not make much difference whether the placebo is small or big and colored or colorless in terms of the strength of the placebo effects; it is usually serious or invasive procedures that generate stronger effects. Injections have been found to present bigger impact than pills. The way in which placebo treatments are administered has been seen to affect the strength of the placebo effect, as well. Additionally, better responses to placebo treatments have been seen in patients who have been administered to by a therapist who has shown both a greater interest in the patient and a greater confidence in the treatment.

Ideas and theories about how placebo treatment actually works, includes operant conditioning, role demands, classical conditioning, hope, guilt reduction, labeling, transference, misattribution, suggestion, control theory, selective symptom monitoring, expectancy effects, cognitive dissonance reduction, endorphin release, anxiety reduction, faith, persuasion.

\section{The Placebo Effect in Control Trials}

The placebo effect is considered to be both a blessing as well as a curse. It is a boon for therapists and medical practitioners because it means the effectiveness of the treatments they prescribe is increased by the psychological nature of the placebo effect. For scientists, on the other hand, the placebo effect can be a problem when trying to assess the effectiveness of medications. For this reason, a type of study known as the placebo controlled, randomized double-blind study was developed to assess medications and discount any placebo effects.

The way these studies work is that volunteers who have signed up to medical trials are placed in different groups, some of these groups being control groups that have alternative or placebo treatment. In addition to the volunteers being placed in different groups, neither the doctor nor the volunteer knows which treatment is being given. If both the patient and the clinician are unaware of the type of treatment being given, this is called double-blind. If the clinician knows which treatment is being given but the patient doesn't, this is called a singleblind trial. Double-blind trials are generally considered to be more reliable because of the risk that the clinician might subconsciously affect the patient's response in single-blind studies.

\section{Evaluating the Placebo Effect}

Despite the research that has been done into the placebo effect and the number of theories put forward to explain it, scientists still don't agree as to how and why the placebo effect works and the way it does. Placebo effect is in some way related to the ancient theory of mind over matter that is, the power of the mind to influence health and well-being works. When a placebo treatment reduces the symptoms of illness, then it is clear that the reduction of symptoms is psychological, although this effect is very pronounced on some than others that makes it a topic to debate.

I hope that upcoming scientists and pharmacists could forward some hypothesis and put an end to this debate that remains inconclusive.
*Corresponding author: Ashish Chauhan, National Institute of Pharmaceutical Education and Research, Mohali, Punjab, India, E-mail: aashishchauhan26@ gmail.com

Received July 30, 2012; Accepted July 31, 2012; Published August 02, 2012

Citation: Chauhan A (2012) A Debatable Drug Trial System. Pharmaceut Ana Acta 3:e116. doi:10.4172/2153-2435.1000e116

Copyright: (c 2012 Chauhan A. This is an open-access article distributed under the terms of the Creative Commons Attribution License, which permits unrestricted use, distribution, and reproduction in any medium, provided the original author and source are credited. 